Jiayu Li $\cdot$ Meng Wang

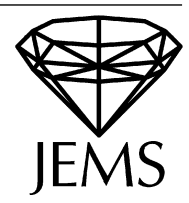

\title{
Liouville theorems for self-similar solutions of heat flows
}

Received April 28, 2006 and in revised form July 23, 2007

Abstract. Let $N$ be a compact Riemannian manifold. A quasi-harmonic sphere on $N$ is a harmonic map from $\left(\mathbb{R}^{m}, e^{-|x|^{2} / 2(m-2)} d s_{0}^{2}\right)$ to $N(m \geq 3)$ with finite energy ([LnW]). Here $d s_{0}^{2}$ is the Euclidean metric in $\mathbb{R}^{m}$. Such maps arise from the blow-up analysis of the heat flow at a singular point. In this paper, we prove some kinds of Liouville theorems for the quasi-harmonic spheres. It is clear that the Liouville theorems imply the existence of the heat flow to the target $N$. We also derive gradient estimates and Liouville theorems for positive quasi-harmonic functions.

Keywords. Harmonic sphere, self-similar solution, quasi-harmonic sphere, heat flow

\section{Introduction}

Let $(M, g)$ and $(N, h)$ be two compact Riemannian manifolds with $\operatorname{dim} M=n$. If a smooth heat flow $u(x, t)$ from $M$ to $N$ blows up at a finite time, we blow up $u$ at a singular point $\left(x_{0}, t_{0}\right)$ by setting $u_{r}(x, t)=u\left(x_{0}+r x, t_{0}+r^{2} t\right)(t<0)$. In [LnW], it is proved that, if there is no harmonic $S^{2}$ on the target $N$, there is a subsequence $r_{k} \rightarrow 0$ such that $u_{r_{k}} \rightarrow u_{\infty}$ strongly in $H_{\mathrm{loc}}^{1}$, where $u_{\infty}$ is a harmonic sphere or a quasi-harmonic sphere, i.e. $u_{\infty}: S^{k} \rightarrow N$ is harmonic, or $u_{\infty}: \mathbb{R}^{m} \times(-\infty, 0) \rightarrow N$ with $u_{\infty}(x, t)=$ $w(x / \sqrt{-t})$, where $w:\left(\mathbb{R}^{m}, e^{-|x|^{2} / 2(m-2)} d s_{0}^{2}\right) \rightarrow N$ is a harmonic map of finite energy $(2 \leq k \leq n-1$ and $3 \leq m \leq n)$. Here $d s_{0}^{2}$ is the Euclidean metric in $\mathbb{R}^{m}$. In other words, $w$ satisfies the equation

$$
\tau(w)=\frac{1}{2} x \cdot \nabla w
$$

with the property that

$$
\int_{\mathbb{R}^{m}}|\nabla w|^{2} e^{-|x|^{2} / 4} d x<\infty
$$

J. Li: Math. Group, The Abdus Salam ICTP, Trieste 34100, Italy, and Academy of Mathematics and Systems Sciences, Chinese Academy of Sciences, Beijing 100080, P.R. China; e-mail: jyli@ ictp.it M. Wang: Department of Mathematics, Zhejiang University, Hangzhou 310027, P.R. China; e-mail: mathdreamcn@yahoo.com.cn

Mathematics Subject Classification (2000): Primary 58E20; Secondary 35 J70 
where

$$
\tau^{k}(w)=\Delta w^{k}+\Gamma_{i j}^{k}(w) \frac{\partial w^{i}}{\partial x^{l}} \frac{\partial w^{j}}{\partial x^{l}}
$$

is the tension field of $w$, and $\Gamma_{i j}^{k}$ are the Christoffel symbols of $N$ in local coordinates.

In a recent paper [DZ], Ding-Zhao showed that equivariant quasi-harmonic spheres are discontinuous at infinity. So the behavior of quasi-harmonic spheres is quite different from that of harmonic spheres.

Furthermore, Lin-Wang [LnW] showed that, if there is no harmonic sphere and no quasi-harmonic sphere on the target $N$, the heat flow is in fact smooth. Therefore, Liouville theorems for harmonic spheres and quasi-harmonic spheres imply global existence of heat flows. In this paper we study Liouville theorems for quasi-harmonic spheres.

Even if $N=\mathbb{R}$, that is, $w$ is a function, the equation 1.1 seems to be new. In this case the equation reduces to a linear equation in $\mathbb{R}^{m}$

$$
\Delta(w)=\frac{1}{2} x \cdot \nabla w .
$$

We can view $w$ as a harmonic function on $\mathbb{R}^{m}$ with metric $d s^{2}=e^{-|x|^{2} / 2(m-2)} \sum_{k=1}^{m} d x_{k}^{2}$. The metric is quite singular at infinity, and it is not complete. One may wonder whether the quasi-harmonic functions still possess the basic properties of harmonic functions. In this paper, we show that there is no nonconstant positive quasi-harmonic function on $\mathbb{R}^{m}$ with polynomial growth, and consequently, there is no nonconstant bounded quasiharmonic function on $\mathbb{R}^{m}$. In general, we derive gradient estimates for positive quasiharmonic functions on $\mathbb{R}^{m}$,

$$
\sup _{B_{R}(0)}|\nabla \log w| \leq C(m) R,
$$

where $C(m)$ depends only on $m$. We show that there is a positive constant $F_{m}$ depending only on $m$ such that any positive quasi-harmonic function on $\mathbb{R}^{m}$ with $\lim _{R \rightarrow \infty} R^{-1} \sup _{B_{R}(0)}|\nabla \log w|<1 / F_{m}$ is constant. In the proof, we use the gradient estimate method developed in [L1] and [L2].

Using gradient estimates for quasi-harmonic spheres, we also show that, if the target manifold is simply connected and complete with nonpositive sectional curvature, there is no nonconstant quasi-harmonic sphere with bounded image.

We say $B_{r}\left(x_{0}\right)$ is a regular ball in $N$ if $\operatorname{Cut}\left(x_{0}\right) \cap B_{r}\left(x_{0}\right)=\emptyset$ and $\sqrt{K} r<\pi / 2$ where $K \geq 0$ is an upper bound of the sectional curvature of $N$ on $B_{r}\left(x_{0}\right)$. The heat flow and harmonic maps into regular balls were studied by Baldes $[\bar{B}]$, Gulliver-Jost [GJ], Hildebrandt [Hi], Hildebrandt-Kaul-Widman [HKW], Jost [J], Li [L] and Li-Wang $[\mathrm{LW}]$. In this paper we show that there is no nonconstant quasi-harmonic sphere with image in a regular ball, which can certainly be applied to the existence of heat flows and harmonic maps into a regular ball.

\section{Nonpositively curved targets}

In this section, we show that, if the target manifold is simply connected and complete with nonpositive sectional curvature, then any quasi-harmonic sphere with bounded image is a 
constant map. This can be seen as a generalization of the classical Liouville theorems for harmonic functions on $\mathbb{R}^{m}$.

Theorem 2.1. Let $N$ be a simply connected complete Riemannian manifold with nonpositively sectional curvature. Let $u$ be a quasi-harmonic map from $\mathbb{R}^{m}$ to $N$, that is, $u$ satisfies the equation (1.1). Assume that $y_{0} \notin u\left(B_{R}(0)\right)$. Let $\rho(y)$ be the distance between $y$ and $y_{0}$ in $N$. Then, if $b>2 \sup \left\{\rho(u(x)) \mid x \in B_{R}(0)\right\}$, we have

$$
\sup _{B_{R / 2}(0)} \frac{|\nabla u|(x)}{b^{2}-\rho^{2}(u(x))} \leq \frac{C}{R b}
$$

where $C>0$ depends only on $m$ and $N$.

Proof. Let

$$
\phi(x)=\frac{|\nabla u|^{2}(x)}{\left(b^{2}-\rho^{2}(u(x))\right)^{2}} .
$$

Then

$$
\nabla \phi(x)=\frac{\nabla\left(|\nabla u|^{2}(x)\right)}{\left(b^{2}-\rho^{2}(u(x))\right)^{2}}+\frac{2 \nabla \rho^{2}|\nabla u|^{2}(x)}{\left(b^{2}-\rho^{2}(u(x))\right)^{3}},
$$

and

$$
\begin{aligned}
\Delta \phi(x)= & \frac{\Delta\left(|\nabla u|^{2}(x)\right)}{\left(b^{2}-\rho^{2}(u(x))\right)^{2}}+\frac{4 \nabla \rho^{2} \nabla|\nabla u|^{2}(x)}{\left(b^{2}-\rho^{2}(u(x))\right)^{3}} \\
& +\frac{2 \Delta \rho^{2}|\nabla u|^{2}(x)}{\left(b^{2}-\rho^{2}(u(x))\right)^{3}}+\frac{6\left|\nabla \rho^{2}\right|^{2}|\nabla u|^{2}(x)}{\left(b^{2}-\rho^{2}(u(x))\right)^{4}} .
\end{aligned}
$$

Note that $(1.1)$ and the Bochner formula (see [EL]) imply

$$
\Delta|\nabla u|^{2} \geq 2|\nabla d u|^{2}+|\nabla u|^{2}+\nabla u \cdot(x \cdot \nabla d u),
$$

and therefore

$$
\begin{aligned}
\Delta \phi(x) \geq & \frac{2|2 \nabla d u|^{2}(x)+|\nabla u|^{2}(x)+\nabla u \cdot(x \cdot \nabla d u)}{\left(b^{2}-\rho^{2}(u(x))\right)^{2}}+\frac{4 \nabla \rho^{2} \nabla|\nabla u|^{2}(x)}{\left(b^{2}-\rho^{2}(u(x))\right)^{3}} \\
& +\frac{2 \Delta \rho^{2}|\nabla u|^{2}(x)}{\left(b^{2}-\rho^{2}(u(x))\right)^{3}}+\frac{6\left|\nabla \rho^{2}\right|^{2}|\nabla u|^{2}(x)}{\left(b^{2}-\rho^{2}(u(x))\right)^{4}} .
\end{aligned}
$$

By (1.1) and the chain rule, we have

$$
\Delta \rho^{2}(u(x))=H\left(\rho^{2}\right)(\nabla u, \nabla u)+\frac{1}{2} x \cdot \nabla \rho^{2}(u(x)),
$$

where $H\left(\rho^{2}\right)$ is the Hessian of $\rho^{2}$. Since the sectional curvature $K_{N}$ of $N$ is nonpositive, the Hessian comparison theorem implies

$$
\Delta \rho^{2}(u(x)) \geq 2|\nabla u|^{2}(x)+\frac{1}{2} x \cdot \nabla \rho^{2}(u(x)) .
$$


Substituting 2.6 into 2.5) yields

$$
\begin{aligned}
\Delta \phi(x) \geq & \frac{2|\nabla d u|^{2}(x)+|\nabla u|^{2}(x)}{\left(b^{2}-\rho^{2}(u(x))\right)^{2}}+\frac{4 \nabla \rho^{2} \nabla|\nabla u|^{2}(x)}{\left(b^{2}-\rho^{2}(u(x))\right)^{3}} \\
& +\frac{4|\nabla u|^{4}(x)}{\left(b^{2}-\rho^{2}(u(x))\right)^{3}}+\frac{6\left|\nabla \rho^{2}\right|^{2}|\nabla u|^{2}(x)}{\left(b^{2}-\rho^{2}(u(x))\right)^{4}} \\
& +\frac{\nabla u \cdot(x \cdot \nabla d u)}{\left(b^{2}-\rho^{2}(u(x))\right)^{2}}+\frac{x \cdot \nabla \rho^{2}(u(x))|\nabla u|^{2}(x)}{\left(b^{2}-\rho^{2}(u(x))\right)^{3}} .
\end{aligned}
$$

It follows from $(2.3)$ that

$$
x \cdot \nabla \phi=\frac{x \cdot \nabla\left(|\nabla u|^{2}(x)\right)}{\left(b^{2}-\rho^{2}(u(x))\right)^{2}}+\frac{2 x \cdot \nabla \rho^{2}|\nabla u|^{2}(x)}{\left(b^{2}-\rho^{2}(u(x))\right)^{3}},
$$

and

$$
\frac{\nabla \rho^{2} \cdot \nabla \phi}{b^{2}-\rho^{2}}=\frac{\nabla \rho^{2} \cdot \nabla\left(|\nabla u|^{2}(x)\right)}{\left(b^{2}-\rho^{2}(u(x))\right)^{3}}+\frac{2\left|\nabla \rho^{2}\right|^{2}|\nabla u|^{2}(x)}{\left(b^{2}-\rho^{2}(u(x))\right)^{4}} .
$$

So

$$
\begin{aligned}
\Delta \phi(x) \geq & \frac{2|\nabla d u|^{2}(x)}{\left(b^{2}-\rho^{2}(u(x))\right)^{2}}++\frac{2 \nabla \rho^{2} \nabla|\nabla u|^{2}(x)}{\left(b^{2}-\rho^{2}(u(x))\right)^{3}} \\
& +\frac{4|\nabla u|^{4}(x)}{\left(b^{2}-\rho^{2}(u(x))\right)^{3}}+\frac{2 \nabla \phi \cdot \nabla \rho^{2}}{b^{2}-\rho^{2}(u(x))} \\
& +\frac{2\left|\nabla \rho^{2}\right|^{2}|\nabla u|^{2}(x)}{\left(b^{2}-\rho^{2}(u(x))\right)^{4}}+\frac{|\nabla u|^{2}(x)}{\left(b^{2}-\rho^{2}(u(x))\right)^{2}}+\frac{1}{2} x \cdot \nabla \phi .
\end{aligned}
$$

Hölder's inequality implies that

$$
\frac{2|\nabla d u|^{2}}{\left(b^{2}-\rho^{2}(u(x))\right)^{2}}+\frac{2\left|\nabla \rho^{2}\right|^{2}|\nabla u|^{2}}{\left(b^{2}-\rho^{2}(u(x))\right)^{4}} \geq 4 \frac{|\nabla d u||\nabla u|\left|\nabla \rho^{2}\right|}{\left(b^{2}-\rho^{2}(u(x))\right)^{3}}
$$

and

$$
\left.|\nabla| \nabla u\right|^{2}|\leq 2| \nabla d u|| \nabla u \mid .
$$

Substituting the last two inequalities into 2.7 we have

$$
\begin{aligned}
\Delta \phi(x) \geq & \frac{4|\nabla u|^{4}}{\left(b^{2}-\rho^{2}(u(x))\right)^{3}}+\frac{|\nabla u|^{2}}{\left(b^{2}-\rho^{2}(u(x))\right)^{2}} \\
& +\frac{2 \nabla \phi \cdot \nabla \rho^{2}}{b^{2}-\rho^{2}(u(x))}+\frac{1}{2} x \cdot \nabla \phi .
\end{aligned}
$$

Let $r(x)=|x|$, and introduce

$$
F(x)=\left(R^{2}-r^{2}(x)\right)^{2} \phi(x) .
$$


Since $\left.F\right|_{\partial B_{R}(0)}=0$, if $\nabla u \not \equiv 0$, then $F$ must achieve its maximum at some point $x_{0}$ in $B_{R}(0)$. Then by the maximum principle we have

$$
\nabla F\left(x_{0}\right)=0
$$

and

$$
\Delta F\left(x_{0}\right) \leq 0
$$

By (2.9) and 2.10) we have, at $x_{0}$,

$$
\frac{\nabla \phi}{\phi}=\frac{4 r \nabla r}{R^{2}-r^{2}}
$$

and

$$
\frac{\Delta \phi}{\phi}-\frac{8 r \nabla r \cdot \nabla \phi}{\left(R^{2}-r^{2}\right) \phi}-\frac{4 m}{R^{2}-r^{2}}+\frac{8 r^{2}}{\left(R^{2}-r^{2}\right)^{2}} \leq 0
$$

It follows that

$$
\frac{\Delta \phi}{\phi}-\frac{24 r^{2}}{\left(R^{2}-r^{2}\right)^{2}}-\frac{4 m}{R^{2}-r^{2}} \leq 0 .
$$

By 2.8, 2.11, 2.12, and 2.13, we have

$4\left(b^{2}-\rho^{2}\right) \phi+\frac{8 r \nabla r \cdot \nabla \rho^{2}}{\left(R^{2}-r^{2}\right)\left(b^{2}-\rho^{2}\right)}+\left(1+\frac{2 r x \cdot \nabla r}{R^{2}-r^{2}}\right)-\frac{24 r^{2}}{\left(R^{2}-r^{2}\right)^{2}}-\frac{4 m}{R^{2}-r^{2}} \leq 0$.

Because

$$
\rho(u(x))<\frac{b}{2}, \quad\left|\nabla \rho^{2}\right| \leq b|\nabla u|, \quad \text { and } \quad \frac{2 r x \cdot \nabla r}{R^{2}-r^{2}}=\frac{2 r^{2}}{R^{2}-r^{2}}>0,
$$

we have

$$
3 b^{2} \phi-\frac{8 r b|\nabla u|}{\left(R^{2}-r^{2}\right)\left(b^{2}-\rho^{2}\right)}-\frac{24 r^{2}}{\left(R^{2}-r^{2}\right)^{2}}-\frac{4 m}{R^{2}-r^{2}} \leq 0 .
$$

Multiplying through 2.14 by $\left(R^{2}-r^{2}\right)^{2}$, we have

$$
3 b^{2} F-8 R b F^{1 / 2}-(24+4 m) R^{2} \leq 0,
$$

which yields

$$
\sup _{B_{R / 2}(0)} F^{1 / 2}(x) \leq F^{1 / 2}\left(x_{0}\right) \leq \frac{C R}{b}
$$

that is,

$$
\sup _{B_{R / 2}(0)} \frac{|\nabla u|(x)}{b^{2}-\rho^{2}(u(x))} \leq \frac{C}{R b} .
$$

This proves the theorem.

The gradient estimate 2.1 clearly implies the following Liouville type theorem.

Theorem 2.2. Let $N$ be a simply connected complete Riemannian manifold with nonpositive sectional curvature. Let $u$ be a quasi-harmonic map from $\mathbb{R}^{m}$ to $N$, that is, u satisfies the equation 1.1. If the image of $u$ in $N$ is a bounded set, then $u$ is constant. 


\section{Image in a regular ball}

Let us first recall the definition of a generalized regular ball from [L] and [LW]. Let $N_{0}$ be a bounded open set of $N$. We say that $N_{0}$ satisfies condition (C) if there is a positive function $f \in C^{2}\left(N_{0}\right)$ satisfying

$$
-\nabla^{2} f-f k_{2}(y) h \geq C_{0}\left(N_{0}\right) h,
$$

and

$$
0<m_{1}\left(N_{0}\right) \leq f(y) \leq m_{2}\left(N_{0}\right)<\infty,
$$

for all $y \in N_{0}$, where

$$
k_{2}(y)=\sup \left\{K(y, \pi) \mid K(y, \pi) \text { is the sectional curvature of a two-plane } \pi \subset T_{y} N\right\},
$$

and $C_{0}\left(N_{0}\right)>0$. If $N_{0}$ satisfies condition (C) and there exists a nonnegative convex function $f^{*}$ on $N_{0}$ such that $N_{0}=\left(f^{*}\right)^{-1}([0, r))$, we call $N_{0}$ a generalized regular ball. It is clear that a regular ball is a generalized regular ball (cf. [L] and [LW]).

Theorem 3.1. Suppose that $N_{0} \subset N$ satisfies condition (C). If $u(x)$ is a quasi-harmonic map from $\mathbb{R}^{m}$ to $N_{0}$, that is, u satisfies the equation [1.1, then

$$
\sup _{B_{R / 2}(0)}|\nabla u| \leq \frac{C_{m} m_{1}}{R},
$$

where $C_{m}$ is a positive constant depending only on $m, C_{0}\left(N_{0}\right), m_{1}\left(N_{0}\right)$ and $m_{2}\left(N_{0}\right)$.

Proof. Set

$$
F(x)=\frac{|\nabla u(x)|^{2}}{f^{2}(u(x))} .
$$

A straightforward computation gives

$$
\nabla F=\frac{\nabla|\nabla u|^{2}}{f^{2}}-\frac{2 \nabla f|\nabla u|^{2}}{f^{3}}
$$

and

$$
\Delta F=\frac{\Delta|\nabla u|^{2}}{f^{2}}-\frac{4 \nabla f \nabla|\nabla u|^{2}}{f^{3}}-\frac{2 \Delta f|\nabla u|^{2}}{f^{3}}+\frac{6|\nabla f|^{2}|\nabla u|^{2}}{f^{4}} .
$$

Note that

$$
\Delta f(u(x))=\nabla^{2}(f)(\nabla u, \nabla u)+\frac{1}{2} x \cdot \nabla f(u(x))
$$

and

$$
\begin{aligned}
\Delta|\nabla u|^{2}= & 2|\nabla d u|^{2}+|\nabla u|^{2}+\nabla u \cdot(x \cdot \nabla d u) \\
& -2 \sum_{i, j=1}^{m}\left\langle R^{N}\left(d u\left(e_{i}\right), d u\left(e_{j}\right)\right) d u\left(e_{i}\right), d u\left(e_{j}\right)\right\rangle,
\end{aligned}
$$


where $e_{1}, \ldots, e_{m}$ is the standard basis of $\mathbb{R}^{m}$, and $R^{N}$ is the curvature operator of $N$. Substituting (3.4), 3.5), and (3.2) into (3.3), using the assumption (C), one gets

$$
\begin{aligned}
\Delta F \geq & \frac{2|\nabla d u|^{2}+|\nabla u|^{2}}{f^{2}}+\frac{2 C_{0}|\nabla u|^{4}}{f^{3}}+\frac{6|\nabla f|^{2}|\nabla u|^{2}}{f^{4}} \\
& +\frac{\nabla u \cdot(x \cdot \nabla d u)}{f^{2}}-\frac{x \cdot \nabla f|\nabla u|^{2}}{f^{3}}-\frac{4 \nabla f \nabla|\nabla u|^{2}}{f^{3}} \\
= & \frac{2|\nabla d u|^{2}+|\nabla u|^{2}}{f^{2}}+\frac{2 C_{0}|\nabla u|^{4}}{f^{3}}+\frac{2|\nabla f|^{2}|\nabla u|^{2}}{f^{4}} \\
& -\frac{2 \nabla f \nabla|\nabla u|^{2}}{f^{3}}-\frac{2 \nabla f \cdot \nabla F}{f}+\frac{1}{2} x \cdot \nabla F
\end{aligned}
$$

By Hölder's inequality, we have

$$
\frac{2|\nabla d u|^{2}}{f^{2}}+\frac{2|\nabla f|^{2}|\nabla u|^{2}}{f^{4}} \geq \frac{4|\nabla d u||\nabla u||\nabla f|}{f^{3}}
$$

and

$$
\left.|\nabla| \nabla u\right|^{2}|\leq 2| \nabla d u|| \nabla u \mid
$$

Substituting the last two inequalities into (3.6), we obtain

$$
\Delta F \geq 2 C_{0} m_{1} F^{2}-2 \nabla F \cdot \frac{\nabla f}{f}+\left(F+\frac{1}{2} x \cdot \nabla F\right) .
$$

Let $r(x)=|x|$, and introduce

$$
\psi(x)=\left(R^{2}-r^{2}(x)\right)^{2} F(x) .
$$

Since $\left.\psi\right|_{\partial B_{R}(0)}=0$, if $\nabla u \neq \equiv 0$, then $\psi$ must achieve its maximum at some point $x_{0}$ in $B_{R}(0)$. Then by the maximum principle we have

$$
\nabla \psi\left(x_{0}\right)=0
$$

and

$$
\Delta \psi\left(x_{0}\right) \leq 0
$$

By 3.8 and 3.9 we have, at $x_{0}$,

$$
\frac{\nabla F}{F}=\frac{4 r \nabla r}{R^{2}-r^{2}}
$$

and

$$
\frac{\Delta F}{F}-\frac{8 r \nabla r \cdot \nabla F}{\left(R^{2}-r^{2}\right) F}-\frac{4 m}{R^{2}-r^{2}}+\frac{8 r^{2}}{\left(R^{2}-r^{2}\right)^{2}} \leq 0 .
$$


It follows that

$$
\frac{\Delta F}{F}-\frac{24 r^{2}}{\left(R^{2}-r^{2}\right)^{2}}-\frac{4 m}{R^{2}-r^{2}} \leq 0 .
$$

By 3.7, 3.10, 3.11 and 3.12, we have

$$
2 C_{0} m_{1} F-\frac{8 R}{R^{2}-r^{2}} F^{1 / 2}+\left(1+\frac{2 r x \cdot \nabla r}{R^{2}-r^{2}}\right)-\frac{24 r^{2}}{\left(R^{2}-r^{2}\right)^{2}}-\frac{4 m}{R^{2}-r^{2}} \leq 0 .
$$

Because

$$
\frac{2 r x \cdot \nabla r}{R^{2}-r^{2}}>0
$$

we have

$$
2 C_{0} m_{1} F-\frac{8 R}{R^{2}-r^{2}} F^{1 / 2}-\frac{24 r^{2}}{\left(R^{2}-r^{2}\right)^{2}}-\frac{4 m}{R^{2}-r^{2}} \leq 0 .
$$

Multiplying through 2.14 by $\left(R^{2}-r^{2}\right)^{2}$, we have

$$
2 C_{0} m_{1} \psi-8 R \psi^{1 / 2}-(24+4 m) R^{2} \leq 0,
$$

which yields

$$
\sup _{B_{R / 2}(0)} \psi^{1 / 2}(x) \leq \psi^{1 / 2}\left(x_{0}\right) \leq C_{m} R
$$

that is,

$$
\sup _{B_{R / 2}(0)} \frac{|\nabla u|(x)}{f(u(x))} \leq \frac{C_{m}}{R}
$$

This proves the theorem.

By the gradient estimate 3.1, we can show the following Liouville type theorem.

Theorem 3.2. Suppose that $N_{0} \subset N$ satisfies condition (C). If $u(x)$ is a quasi-harmonic map from $\mathbb{R}^{m}$ to $N_{0}$, that is, u satisfies the equation 1.1] with image in $N_{0}$, then $u$ is constant.

\section{Positive functions}

In this section, we consider the positive quasi-harmonic functions on $\mathbb{R}^{m}$.

Theorem 4.1. Let $u$ be a positive quasi-harmonic function on $\mathbb{R}^{m}$, that is, $u>0$ satisfies the equation (1.3). Then we have the gradient estimate

$$
\sup _{B_{R}(0)}|\nabla \log u| \leq C(m) R
$$

where $C(m)$ is a positive constant depending only on $m$. There is a positive constant $F_{m}>0$ such that, if in addition

$$
\lim _{R \rightarrow \infty} R^{-1} \sup _{B_{R}(0)}|\nabla \log u|<\frac{1}{F_{m}}
$$

then $u$ is a constant. 
Proof. Let $\omega=u^{-\beta}$, where $0<\beta<1$ is to be defined later. Then

$$
\nabla \omega=-\beta u^{-\beta-1} \nabla u, \quad \frac{|\nabla \omega|}{\omega}=\beta \frac{|\nabla u|}{u}, \quad \Delta \omega=\frac{\beta+1}{\beta} \frac{|\nabla \omega|^{2}}{\omega}+\frac{1}{2} x \cdot \nabla \omega .
$$

Let $\phi(x)=|\nabla \omega|^{2} / \omega^{2}$. Then

$$
\begin{aligned}
& \nabla \phi(x)=\frac{\nabla\left(|\nabla \omega|^{2}\right)}{\omega^{2}}-2 \frac{|\nabla \omega|^{2} \nabla \omega}{\omega^{3}}, \\
& \Delta \phi(x)=\frac{\Delta\left(|\nabla \omega|^{2}\right)}{\omega^{2}}-4 \frac{\nabla \omega \cdot \nabla\left(|\nabla \omega|^{2}\right)}{\omega^{3}}+6 \frac{|\nabla \omega|^{4}}{\omega^{4}}-2 \frac{|\nabla \omega|^{2} \Delta \omega}{\omega^{3}} .
\end{aligned}
$$

Note that

$\Delta\left(|\nabla \omega|^{2}\right)=2|\nabla d \omega|^{2}+2 \frac{\beta+1}{\beta} \frac{\nabla \omega \cdot \nabla\left(|\nabla \omega|^{2}\right)}{\omega}-2 \frac{\beta+1}{\beta} \frac{|\nabla \omega|^{4}}{\omega^{2}}+|\nabla \omega|^{2}+\sum_{k, i} \omega_{i} x_{k} \omega_{k i}$.

Then

$$
\begin{aligned}
\Delta \phi(x)= & \frac{2|\nabla d \omega|^{2}}{\omega^{2}}+\left(2 \frac{\beta+1}{\beta}-4\right) \frac{\nabla \omega \cdot \nabla\left(|\nabla \omega|^{2}\right)}{\omega^{3}}+\left(6-2 \frac{\beta+1}{\beta}\right) \frac{|\nabla \omega|^{4}}{\omega^{4}} \\
& +\frac{|\nabla \omega|^{2}}{\omega^{2}}+\frac{\sum_{k, i} \omega_{i} x_{k} \omega_{k i}}{\omega^{2}}-2 \frac{|\nabla \omega|^{2}}{\omega^{3}}\left[\frac{\beta+1}{\beta} \frac{|\nabla \omega|^{2}}{\omega}+\frac{1}{2} x \cdot \nabla \omega\right] \\
= & \frac{2|\nabla d \omega|^{2}}{\omega^{4}}+\left(2-\frac{4}{\beta}\right) \frac{|\nabla \omega|^{4}}{\omega^{4}}+\phi \\
& +\left[\frac{\sum_{k, i} \omega_{i} x_{k} \omega_{k i}}{\omega^{2}}-\frac{|\nabla \omega|^{2} x \cdot \nabla \omega}{\omega^{3}}\right]+\frac{2(1-\beta)}{\beta}\left[\frac{\nabla \omega \cdot \nabla \phi}{\omega}+2 \frac{|\nabla \omega|^{4}}{\omega^{4}}\right] \\
= & \frac{2|\nabla d \omega|^{2}}{\omega^{2}}-2 \frac{|\nabla \omega|^{4}}{\omega^{4}}+\phi+\frac{1}{2} x \cdot \nabla \phi+\frac{2(1-\beta)}{\beta} \frac{\nabla \omega \cdot \nabla \phi}{\omega} .
\end{aligned}
$$

By Cauchy's inequality, we have

$$
|\nabla d \omega|^{2} \geq \frac{1}{m}(\Delta \omega)^{2}
$$

therefore

$$
\frac{|\nabla d \omega|^{2}}{\omega^{2}} \geq \frac{1}{m} \frac{(\beta+1)^{2}}{\beta^{2}} \frac{|\nabla \omega|^{4}}{\omega^{4}}+\frac{1}{4 m} \frac{|x \cdot \nabla \omega|^{2}}{\omega^{2}}+\frac{\beta+1}{m \beta} \frac{|\nabla \omega|^{2}}{\omega^{3}} x \cdot \nabla \omega .
$$

By Hölder's inequality, we get

$$
\frac{|\nabla d \omega|^{2}}{\omega^{2}} \geq\left(\frac{1}{m} \frac{(\beta+1)^{2}}{\beta^{2}}-1\right) \frac{|\nabla \omega|^{4}}{\omega^{4}}+\left(\frac{1}{4 m}-\frac{(\beta+1)^{2}}{(2 m \beta)^{2}}\right) \frac{|x \cdot \nabla \omega|^{2}}{\omega^{2}} .
$$


Substituting the last inequality into 4.1, we obtain

$$
\begin{aligned}
\Delta \phi(x) \geq & \left(\frac{2}{m} \frac{(\beta+1)^{2}}{\beta^{2}}-4\right) \frac{|\nabla \omega|^{4}}{\omega^{4}}+\phi+\frac{1}{2} x \cdot \nabla \phi+\frac{2(1-\beta)}{\beta} \frac{\nabla \omega \cdot \nabla \phi}{\omega} \\
& +2\left(\frac{1}{4 m}-\frac{(\beta+1)^{2}}{(2 m \beta)^{2}}\right) \frac{|x \cdot \nabla \omega|^{2}}{\omega^{2}} .
\end{aligned}
$$

We choose $0<\beta<1$ such that

$$
\frac{2}{m} \frac{(\beta+1)^{2}}{\beta^{2}}-4=1 .
$$

Then from 4.2 we have

$$
\Delta \phi(x) \geq|\phi(x)|^{2}+A_{m} x \cdot \nabla \phi+B_{m} \frac{\nabla \omega \cdot \nabla \phi}{\omega}-C_{m}|x|^{2}|\phi(x)| .
$$

Using (4.1) and Hölder's inequality, we can have another estimate for $\Delta \phi(x)$ :

$$
\begin{aligned}
\Delta \phi(x)= & \frac{2|\nabla d \omega|^{2}}{\omega^{4}}+\left(2-\frac{4}{\beta}\right) \frac{|\nabla \omega|^{4}}{\omega^{4}}-2 \frac{\nabla \omega \cdot \nabla\left(|\nabla \omega|^{2}\right)}{\omega^{3}}+\phi \\
& +\frac{1}{2} x \cdot \nabla \phi+\frac{2}{\beta}\left[\frac{\nabla \omega \cdot \nabla \phi}{\omega}+2 \frac{|\nabla \omega|^{4}}{\omega^{4}}\right] \\
\geq & \phi+\frac{1}{2} x \cdot \nabla \phi+\frac{2}{\beta} \frac{\nabla \omega \cdot \nabla \phi}{\omega} .
\end{aligned}
$$

Let $F(x)=\left[R^{2}-r^{2}(x)\right]^{2} \phi(x)=\left[R^{2}-r^{2}(x)\right]^{2}|\nabla \omega|^{2} / \omega^{2}$. Suppose that $x_{0}$ is the maximal point on $\overline{B_{R}(0)}$. If $\nabla \omega \not \equiv 0$ then $x_{0} \in B_{R}(0)$. Thus at $x_{0}$,

$$
\nabla F=0
$$

and

$$
\Delta F \leq 0
$$

From 4.5) and 4.6,

$$
\begin{gathered}
\frac{\nabla \phi}{\phi}=\frac{4 r \nabla r}{R^{2}-r^{2}}, \\
\frac{\Delta \phi}{\phi}-\frac{8 r \nabla r \cdot \nabla \phi}{\left(R^{2}-r^{2}\right) \phi}-\frac{4 m}{R^{2}-r^{2}}+\frac{8 r^{2}}{\left(R^{2}-r^{2}\right)^{2}} \leq 0 .
\end{gathered}
$$

Then

$$
\frac{\Delta \phi}{\phi}-\frac{24 r^{2}}{\left(R^{2}-r^{2}\right)^{2}}-\frac{4 m}{\left(R^{2}-r^{2}\right)} \leq 0 .
$$


Using the same argument as in Section 3, by 4.3 and the above inequality, we have at $x_{0}$,

$$
\phi-A_{m} \frac{r^{2}}{R^{2}-r^{2}}-B_{m} \frac{4 r}{R^{2}-r^{2}} \frac{|\nabla \omega|}{\omega} \omega-C_{m} r^{2}-\frac{24 r^{2}}{\left(R^{2}-r^{2}\right)^{2}}-\frac{4 m}{R^{2}-r^{2}} \leq 0 .
$$

Multiplying through 4.7 by $\left(R^{2}-r^{2}\right)^{2}$, we have

$$
F\left(x_{0}\right)-4 B_{m} R F^{1 / 2}\left(x_{0}\right)-D_{m}\left(R^{6}+1\right) \leq 0,
$$

which implies that

$$
\sup _{B_{R / 2}(0)} \frac{|\nabla u|}{u} \leq E_{m} R
$$

This proves the first part of the theorem. Instead of using (4.3), we now use (4.4); by an argument similar to the one used in obtaining 4.7, we can get

$$
1+\frac{2 r^{2}}{R^{2}-r^{2}}-2 \frac{4 r}{R^{2}-r^{2}} \frac{|\nabla u|}{u} \leq \frac{24 r^{2}}{\left(R^{2}-r^{2}\right)^{2}}+\frac{4 m}{\left(R^{2}-r^{2}\right)} .
$$

Multiplying through the last inequality by $R^{2}-r^{2}$, we have

$$
R^{2}+r^{2} \leq F_{m} R \sup _{B_{R}(0)}|\nabla \log u|+\frac{24 r^{2}}{R^{2}-r^{2}}+4 m
$$

thus,

$$
R^{2} \leq F_{m} R \sup _{B_{R}(0)}|\nabla \log u|+\frac{r^{4}-\left(R^{2}-24\right) r^{2}}{R^{2}-r^{2}}+4 m .
$$

It is clear that we may assume that at the maximum point $x_{0}$ of $F, r^{2}\left(x_{0}\right) \leq R^{2}-24$, because of 4.8. If $\lim _{R \rightarrow \infty} R^{-1} \sup _{B_{R}(0)}|\nabla \log u|<1 / F_{m}$, letting $R \rightarrow \infty$, we get a contradiction, which implies that $|\nabla u| \equiv 0$. This proves the theorem.

Theorem 4.2. Let $u$ be a positive quasi-harmonic function on $\mathbb{R}^{m}$, that is, $u$ satisfies the equation (1.3). If $\sup _{B_{R}(0)} u(x) \leq C P(R)$, where $P(t)$ is a polynomial of $t$, then $u$ is a constant.

Proof. Without loss of generality, we may assume that $u(x) \geq \delta>0$. Otherwise, we consider $u+\delta$ instead of $u$. Let $\omega=u^{-\beta}$. Then

$$
\nabla \omega=-\beta u^{-\beta-1} \nabla u, \quad \Delta \omega=\frac{\beta+1}{\beta} \frac{|\nabla \omega|^{2}}{\omega}+\frac{1}{2} x \cdot \nabla \omega .
$$

Let $f(R)=\sup _{B_{R}(0)} u(x)$. Then

$$
\inf _{x \in B_{R}(0)} \omega(x) \geq f^{-\beta}(R) .
$$


Let $\phi(x)=|\nabla \omega|^{2} / \omega^{4}$. Then

$$
\begin{aligned}
& \nabla \phi(x)=\frac{\nabla\left(|\nabla \omega|^{2}\right)}{\omega^{4}}-4 \frac{|\nabla \omega|^{2} \nabla \omega}{\omega^{5}}, \\
& \Delta \phi(x)=\frac{\Delta\left(|\nabla \omega|^{2}\right)}{\omega^{4}}-8 \frac{\nabla \omega \cdot \nabla\left(|\nabla \omega|^{2}\right)}{\omega^{5}}+20 \frac{|\nabla \omega|^{4}}{\omega^{6}}-4 \frac{|\nabla \omega|^{2} \Delta \omega}{\omega^{5}} .
\end{aligned}
$$

Note that

$$
\Delta\left(|\nabla \omega|^{2}\right)=2|\nabla d \omega|^{2}+2 \frac{\beta+1}{\beta} \frac{\nabla \omega \cdot \nabla\left(|\nabla \omega|^{2}\right)}{\omega}-2 \frac{\beta+1}{\beta} \frac{|\nabla \omega|^{4}}{\omega^{2}}+|\nabla \omega|^{2}+\sum_{k, i} \omega_{i} x_{k} \omega_{k i} .
$$

Then

$$
\begin{aligned}
\Delta \phi(x)= & \frac{2|\nabla d \omega|^{2}}{\omega^{4}}+\left(2 \frac{\beta+1}{\beta}-8\right) \frac{\nabla \omega \cdot \nabla\left(|\nabla \omega|^{2}\right)}{\omega^{5}}+\left(20-2 \frac{\beta+1}{\beta}\right) \frac{|\nabla \omega|^{4}}{\omega^{6}} \\
& +\frac{|\nabla \omega|^{2}}{\omega^{4}}+\frac{\sum_{k, i} \omega_{i} x_{k} \omega_{k i}}{\omega^{4}}-4 \frac{|\nabla \omega|^{2}}{\omega^{5}}\left[\frac{\beta+1}{\beta} \frac{|\nabla \omega|^{2}}{\omega}+\frac{1}{2} x \cdot \nabla \omega\right] \\
= & \frac{2|\nabla d \omega|^{2}}{\omega^{4}}+\frac{2(1-3 \beta)}{\beta} \varepsilon \frac{\nabla \omega \cdot \nabla\left(|\nabla \omega|^{2}\right)}{\omega^{5}}+\left(14-\frac{6}{\beta}\right) \frac{|\nabla \omega|^{4}}{\omega^{6}}+\phi \\
& +\left[\frac{\sum_{k, i} \omega_{i} x_{k} \omega_{k i}}{\omega^{4}}-2 \frac{|\nabla \omega|^{2} x \cdot \nabla \omega}{\omega^{5}}\right] \\
& +\frac{2(1-3 \beta)}{\beta}(1-\varepsilon)\left[\frac{\nabla \omega \cdot \nabla \phi}{\omega}+4 \frac{|\nabla \omega|^{4}}{\omega^{6}}\right] \\
= & \frac{2|\nabla d \omega|^{2}}{\omega^{4}+\frac{2(1-3 \beta)}{\beta} \varepsilon \frac{\nabla \omega \cdot \nabla\left(|\nabla \omega|^{2}\right)}{\omega^{5}}} \\
& +\left(14-\frac{6}{\beta}+\frac{8}{\beta}(1-3 \beta)(1-\varepsilon)\right) \frac{|\nabla \omega|^{4}}{\omega^{6}} \\
& +\phi+\frac{1}{2} x \cdot \nabla \phi+\frac{2(1-3 \beta)(1-\varepsilon)}{\beta} \frac{\nabla \omega \cdot \nabla \phi}{\omega} \\
\geq & {\left[14-\frac{6}{\beta}+\frac{8}{\beta}(1-3 \beta)(1-\varepsilon)-\frac{2 \varepsilon^{2}(1-3 \beta)^{2}}{\beta^{2}}\right] \frac{|\nabla \omega|^{4}}{\omega^{6}} } \\
& +\frac{2(1-3 \beta)(1-\varepsilon)}{\beta} \frac{\nabla \omega \cdot \nabla \phi}{\omega}+\phi+\frac{1}{2} x \cdot \nabla \phi \\
= & A_{\beta, \varepsilon} \omega^{2} \phi^{2}+B_{\beta, \varepsilon} \frac{\nabla \omega \cdot \nabla \phi}{\omega}+\phi+\frac{1}{2} x \cdot \nabla \phi,
\end{aligned}
$$

where $0<\varepsilon<1$ will be determined later and

$$
\begin{aligned}
A_{\beta, \varepsilon} & =14-\frac{6}{\beta}+\frac{8}{\beta}(1-3 \beta)(1-\varepsilon)-\frac{2 \varepsilon^{2}(1-3 \beta)^{2}}{\beta^{2}} \\
& =-\frac{2}{\beta^{2}}\left[\left(9 \varepsilon^{2}-12 \varepsilon+5\right) \beta^{2}-\left(6 \varepsilon^{2}-4 \varepsilon+1\right) \beta+\varepsilon^{2}\right],
\end{aligned}
$$


and

$$
B_{\beta, \varepsilon}=\frac{2(1-3 \beta)(1-\varepsilon)}{\beta}
$$

Since for all $\varepsilon \in \mathbb{R}$,

$$
9 \varepsilon^{2}-12 \varepsilon+5>0
$$

and

$$
\begin{aligned}
\Delta & =\left(6 \varepsilon^{2}-4 \varepsilon+1\right)^{2}-4 \varepsilon^{2}\left(9 \varepsilon^{2}-12 \varepsilon+5\right) \\
& =8 \varepsilon^{2}-8 \varepsilon+1>0 \quad \text { if } \varepsilon<(2-\sqrt{2}) / 4
\end{aligned}
$$

we have

$$
A_{\beta, \varepsilon}=-\frac{2}{\beta^{2}}\left[\left(9 \varepsilon^{2}-12 \varepsilon+5\right) \beta^{2}-\left(6 \varepsilon^{2}-4 \varepsilon+1\right) \beta+\varepsilon^{2}\right]>0
$$

when

$$
\varepsilon<(2-\sqrt{2}) / 4
$$

and

$$
0<\frac{6 \varepsilon^{2}-4 \varepsilon+1-\sqrt{8 \varepsilon^{2}-8 \varepsilon+1}}{2\left(9 \varepsilon^{2}-12 \varepsilon+5\right)}<\beta<\frac{6 \varepsilon^{2}-4 \varepsilon+1+\sqrt{8 \varepsilon^{2}-8 \varepsilon+1}}{2\left(9 \varepsilon^{2}-12 \varepsilon+5\right)} .
$$

We conclude that

$$
\Delta \phi \geq A_{\beta, \varepsilon} f^{-2 \beta}(R) \phi^{2}+B_{\beta, \varepsilon} \frac{\nabla \omega \cdot \nabla \phi}{\omega}+\phi+\frac{1}{2} x \cdot \nabla \phi .
$$

Let $F(x)=\left[R^{2}-r^{2}(x)\right]^{2} \phi(x)=\left[R^{2}-r^{2}(x)\right]^{2}|\nabla \omega|^{2} / \omega^{4}$. Suppose that $F(x)$ achieves its maximum at $x_{0} \in \overline{B_{R}(0)}$. If $\nabla \omega \not \equiv 0$, then $x_{0} \in B_{R}(0)$. Thus at $x_{0}$, we have

$$
\begin{gathered}
\nabla F=0, \\
\Delta F \leq 0 .
\end{gathered}
$$

From 4.12 and 4.13

$$
\frac{\nabla \phi}{\phi}=\frac{4 r \nabla r}{R^{2}-r^{2}}, \quad \frac{\Delta \phi}{\phi}-\frac{8 r \nabla r \cdot \nabla \phi}{\left(R^{2}-r^{2}\right) \phi}-\frac{4 m}{R^{2}-r^{2}}+\frac{8 r^{2}}{\left(R^{2}-r^{2}\right)^{2}} \leq 0 .
$$

Then

$$
\frac{\Delta \phi}{\phi}-\frac{24 r^{2}}{\left(R^{2}-r^{2}\right)^{2}}-\frac{4 m}{R^{2}-r^{2}} \leq 0 .
$$

By (4.9) and (4.10), we see that $\beta$ can be sufficiently small for $\varepsilon$ small enough. So we can choose $\beta>0$ and $\varepsilon>0$ such that $B_{\beta, \varepsilon}>0$. By the same argument as in Section 3, using (4.11) and the above inequality, we have at $x_{0}$,

$$
A_{\beta, \varepsilon} f^{-2 \beta}(R) \phi-B_{\beta, \varepsilon} \frac{4 R}{R^{2}-r^{2}} \frac{|\nabla \omega|}{\omega^{2}} \omega-\frac{24 r^{2}}{\left(R^{2}-r^{2}\right)^{2}}-\frac{4 m}{R^{2}-r^{2}} \leq 0 .
$$


Multiplying through 4.14 by $\left(R^{2}-r^{2}\right)^{2}$, we have

$$
A_{\beta, \varepsilon} f^{-2 \beta}(R) F\left(x_{0}\right)-4 B_{\beta, \varepsilon} R \delta^{-\beta} F^{1 / 2}\left(x_{0}\right)-(24+4 m) R^{2} \leq 0 .
$$

Then

$$
F^{1 / 2}\left(x_{0}\right) \leq \frac{4 B_{\beta, \varepsilon} R \delta^{-\beta}+\sqrt{16 B_{\beta, \varepsilon}^{2} R^{2} \delta^{-2 \beta}+4(24+4 m) R^{2} A_{\beta, \varepsilon} f^{-2 \beta}(R)}}{2 A_{\beta, \varepsilon} f^{-2 \beta}(R)} .
$$

Note that

$$
\sup _{B_{R / 2}(0)} \frac{|\nabla \omega|}{\omega^{2}}=\beta \sup _{B_{R / 2}(0)} u^{\beta} \frac{|\nabla u|}{u} \geq \beta \delta^{\beta} \sup _{B_{R / 2}(0)} \frac{|\nabla u|}{u} .
$$

By 4.15) and 4.16,

$$
\begin{aligned}
\sup _{B_{R / 2}(0)} \frac{|\nabla u|}{u} & \leq \frac{1}{\beta R} \cdot \frac{2 B_{\beta, \varepsilon} \delta^{-2 \beta}+\delta^{-\beta} \sqrt{4 B_{\beta, \varepsilon}^{2} \delta^{-2 \beta}+(24+4 m) A_{\beta, \varepsilon} f^{-2 \beta}(R)}}{A_{\beta, \varepsilon} f^{-2 \beta}(R)} \\
& =C_{\beta, \varepsilon} \frac{f^{2 \beta}(R)}{\delta^{2 \beta} R} .
\end{aligned}
$$

Here $\beta$ and $\varepsilon$ satisfy (4.9) and (4.10), from which we know that $\beta$ can be sufficiently small for $\varepsilon$ small enough. If there exists a constant $N_{0}$ such that $f(R) \leq R^{N_{0}}$, we can choose $0<\beta<1 / 2 N_{0}$ so that

$$
\sup _{B_{R / 2}(0)} \frac{|\nabla u|}{u} \leq C_{\beta, \varepsilon} \frac{R^{2 \beta N_{0}}}{\delta^{2 \beta} R} .
$$

Leting $R \rightarrow \infty$, we have $|\nabla u| \equiv 0$. This proves the theorem.

Acknowledgments. This work was essentially carried out when M. Wang visited the Abdus Salam ICTP. She thanks the center for their hospitality. The authors thank the referee for his helpful comments.

The research was partially supported by NSFC 10701064 .

\section{References}

[B] Baldes, A.: Harmonic mappings with a partially free boundary. Manuscripta Math. 40, 255-275 (1982) Zbl 0504.58016 MR 0683042

[DZ] Ding, W., Zhao, Y.: Elliptic equations strongly degenerate at a point. Nonlinear Anal. 65, 1624-1632 (2006) Zbl pre05047625 MR 2248689

[EL] Eells, J., Lemaire, L.: Selected Topics in Harmonic Maps. CBMS Reg. Conf. Ser. Math. 50, Amer. Math. Soc. (1983) Zbl 0515.58011 MR 0703510

[GJ] Gulliver, R., Jost, J.: Harmonic maps which solve a free-boundary problem. J. Reine Angew. Math. 38, 61-89 (1987) Zbl 0619.35117 MR 0918841 
[Hi] Hildebrandt, S.: Harmonic mappings of Riemannian manifolds. In: Harmonic Maps and Minimal Immersions (Montecatini, 1984), Lecture Notes in Math. 1161, Springer, Berlin, 1-117 (1985) Zbl 0581.58011 MR 0821968

[HKW] Hildebrandt, S., Kaul, H., Widman, K.-O.: An existence theorem for harmonic mappings of Riemannian manifolds. Acta Math. 138, 1-16 (1977) Zbl 0356.53015 MR 0433502

[J] Jin, Z.: Liouville theorems for harmonic maps. Invent. Math. 108, 1-10 (1992) Zbl 0768.53016 MR 1156381

[L] Li, J.: Heat flows and harmonic maps with a free boundary. Math. Z. 217, 487-495 (1994) Zbl 0821.58012 MR 1306674

[L1] Li, J.: Gradient estimates and Harnack inequalities for nonlinear parabolic and nonlinear elliptic equations on Riemannian manifolds. J. Funct. Anal. 100, 233-256 (1991) Zbl 0746.58078 MR 1125225

[L2] Li, J.: Gradient estimate for the heat kernel of a complete Riemannian manifold and its applications. J. Funct. Anal. 97, 293-310 (1991) Zbl 0724.58064 MR 1111183

[LW] Li, J., Wang, S.: The heat flows and harmonic maps from complete manifolds into regular balls. Bull. Austral. Math. Soc. 58, 177-187 (1998) Zbl 0922.58016 MR 1642183

[LnW] Lin, F. H., Wang, C. Y.: Harmonic and quasi-harmonic spheres. Comm. Anal. Geom. 7, 397-429 (1999) Zbl 0934.58018 MR 1685578 